\title{
Expression of the scaffold connector enhancer of kinase suppressor of Ras 1 (CNKSR1) is correlated with clinical outcome in pancreatic cancer
}

Humair S. Quadri ${ }^{1 \dagger}$, Taylor J. Aiken ${ }^{1+}$, Michael Allgaeuer $^{2 \dagger}$, Radim Moravec ${ }^{3}$, Sean Altekruse ${ }^{3}$, S. Perwez Hussain ${ }^{4}$, Markku M. Miettinen², Stephen M. Hewitt ${ }^{5}$ and Udo Rudloff ${ }^{*}$

\begin{abstract}
Background: Despite the near universal occurrence of activating codon 12 KRAS somatic variants in pancreatic cancer, there is considerable heterogeneity in the molecular make-up, MAPK/ERK pathway activation states, and clinical outcome in this disease. We analyzed the expression levels of CNKSR1, a scaffold that influences MAPK ERK pathway activity, in clinical pancreas cancer specimens and their impact on survival of patients with pancreatic cancer.

Methods: Immunohistochemical staining for CNKSR1 expression was performed on 120 specimens from three independent pancreatic cancer tissue registries, phospho-ERK levels were measured in 86 samples. Expression was divided into CNKSR1 low and CNKSR1 high and correlated with clinicopathological variables including overall survival using multivariate Cox proportional hazard ratio models.

Results: CNKSR1 expression was increased in tumors compared to matched normal uninvolved resection specimens ( $p=0.004)$. 28.3\% (34/120) of patient specimens stained as CNKSR1 low compared to 71.7\% (86/120) of specimens which stained as CNKSR1 high. High CNKSR1 expression was more prevalent in low grade tumors $(p=0.04)$. In multivariate analysis, low CNKSR1 expression status was independently correlated with decreased overall survival ( $H R=2.146 ; 95 \% \mathrm{Cl} 1.34$ to 3.43$)$. When stratifying primary, non-metastatic tumor biopsies by CNKSR1 expression, resection was associated with improved survival in patients with high CNKSR1 expression $(p<0.0001)$ but not low CNKSR1 expression $(p=0.3666)$. Pancreatic tumors with nuclear in addition to cytoplasmic CNKSR1 staining (32/107) showed increased nuclear phospho-ERK expression compared to tumor with cytoplasmic CNKSR1 staining only $(p=0.017)$.

Conclusion: CNKSR1 expression is increased in pancreatic tissue specimens and was found to be an independent prognostic marker of overall survival. CNKSR1 may help to identify patient subgroups with unfavorable tumor biology in order to improve risk stratification and treatment selection. Cellular distribution of CNKSR1 was correlated with nuclear pERK expression.
\end{abstract}

Keywords: Pancreas cancer, Biomarker, Correlative tissue study, Scaffold connector enhancer of kinase suppressor of Ras 1 (CNKSR1)

\footnotetext{
* Correspondence: rudloffu@mail.nih.gov

${ }^{\dagger}$ Equal contributors

${ }^{1}$ Thoracic and Gastrointestinal Oncology Branch, Gastrointestinal Oncology

Section, Investigator Center for Cancer Research, National Cancer Institute,

Building 10 - Hatfield CRC, Room 4-5950, Bethesda, MD 20892, USA

Full list of author information is available at the end of the article
} 


\section{Background}

While advances in the understanding of cancer biology, screening and risk-reducing interventions, and improved treatments have significantly reduced cancer mortality overall, pancreatic cancer remains a deadly disease. The American Cancer Society estimates 53,070 new cases and 41,780 deaths from pancreatic cancer in the United States during 2016 and predicts that pancreatic cancer will rank second of all cancer-related mortalities by the year 2030 [1, 2]. Neither current chemotherapy nor molecular therapy provides patients with an extension of survival measured by more than a few months, or the hope for sustained tumor regressions. Even in the minority of patients who are able to undergo surgical resection, median overall survival remains poor [3]. To date, only a few biomarkers have been associated with survival outcomes in pancreatic cancer [3, 4]. Considering the variability of clinical outcome and the uncertainty of the role of surgical resection in cancers at high risk for early progression, prognostic biomarkers accurately stratifying patients for individualized clinical decision-making would fill an unmet clinical need.

Activating somatic KRAS mutations are nearly omnipresent and a hallmark in the genetic make-up of pancreatic ductal adenocarcinoma (PDAC) [5]. While KRAS mutations themselves have been associated as prognostic markers, there is considerable and significant heterogeneity in the activation states of the downstream MAPK/ ERK pathway, the molecular landscape, response to therapy, and clinical outcome across pancreatic cancers [6-9]. The MAPK/ERK (MEK) pathway downstream of RAS has been the topic of significant research efforts in attempting to target and inhibit the oncogenic progression of RAS-mutant tumors. Certain scaffolding kinase proteins are essential to the spatiotemporal regulation of MAPK/ERK pathway signaling, as well as for regulating and integrating input from and output to other key signaling pathways involved in cellular homeostasis $[10,11]$. The Kinase Suppressor of Ras-1 (KSR1) is a well-examined scaffolding protein; it has been shown to mediate tumor progression in pancreas cancer and may govern response to treatment $[12,13]$. For example, KSR1 can compete with binding partners of BRAF and directly modulate response to small molecule inhibition of the MAPK pathway at the RAF level and it has been shown to be dysregulated in endometrial and colon cancers [14-16]. CNKSR1 (connector enhancer of the Kinase Suppressor of Ras-1), a regulator and binding partner of KSR1, is another scaffolding protein which is less understood. Its role in pancreatic cancer biology, or as a biomarker, remains to be explored.

Current data suggests that CNKSR1 has multiple roles cancer biology, with some reports demonstrating that CNKSR1 interacts with tumor suppressors and others describe its scaffolding protein interactions as oncogenic $[17,18]$. These include, for example, connecting Ephrin B stimulation via small GTPases to c-Jun N-terminal kinase (JNK) activation resulting in net cancer cell migration, or promoting cancer cell proliferation through the Akt-FoxO signalling axis $[19,20]$. Recent studies using phosphomimetic mutants of CNKSR1 have identified phosphorylation sites in the scaffold critical for nuclear translocation and activation of MAPK pathway genes [21]. However, to date all CNKSR1 analysis in the context of pancreatic cancer has been performed at a molecular level with no translational or clinically oriented application. Using pancreatic tumor tissues from three independent cohorts, we aimed to evaluate the expression levels of CNKSR1 and its association with clinicopathological parameters and survival in pancreatic cancer. In addition we aimed to assess the association of CNKSR1 expression levels with MAPK pathway activity as measured by phospho-ERK. The observed association of CNKSR1 expression and survival outcome suggests scaffolding proteins of the RAS-MAPK pathway may account, in part, for the observed heterogeneity of PDAC biology, and clinically may aid in improved future patient stratification.

\section{Methods \\ Study participants and tissue microarray (TMA) composition}

De-identified cancer tissues included in this analysis were confirmed to be pancreatic ductal adenocarcinomas based on pathology slide review at the National Cancer Institute. The analytic dataset included 120 cases, including 99 from the Iowa, Hawaii and Los Angeles Surveillance, Epidemiology, and End Results (SEER) Residual Tumor Registries pancreatic cancer tissue microarray (TMA) [22]. Another 18 cases were patients treated at the University of Maryland at Baltimore Hospital (Baltimore, MD) and three more patients who were treated at the Clinical Center of the National Institutes of Health (Bethesda, MD). Appropriate ethical and transfer of material approvals were obtained from originating sites, as well as the NCI. A commercially purchased TMA of 71 cases (U.S. BioMax, Inc., Rockeville, MD) and a PDAC TMA from 47 patients treated at Stony Brook University (Stony Brook, NY) were used as a secondary cohort to confirm similar CNKSR1 expression distributions.

\section{Case attributes}

The analytic dataset included demographic and clinical data, enabling analyses of CNKSR1 expression by age, race, gender, grade, resection status, the TNM variables lymph node status (N0; No regional lymph node metastasis, N1; Regional lymph node metastasis, and NX; 
Regional lymph nodes cannot be assessed) and distant metastasis (M0; No distant metastasis, and M1; Distant metastasis), SEER stage (localized, regional, and distant), radiation, and primary tumor location. Grade of tumor differentiation was determined upon initial diagnostic workup by the primary pathologist and derived from original pathology reports and data available within the SEER tumor registry. Histologic grade was based on overall extent of glandular differentiation within the resected specimen and, with the limitations of reviewing small tissue cores on a TMA, was re-confirmed in select cases. No re-classifications of the original grading upon re-review were made. Of the different grading systems the WHO 2010 [WHO Classification] classification was used defining Grade 1 as well differentiated ( $>95 \%$ of tumor composed of glands), Grade 2 moderately differentiated ( $50 \%$ - $95 \%$ glands), and grade 3 poorly differentiated $(<50 \%$ glands) [23]. Person months from diagnosis to date of last follow-up or death were recorded. Time contributed by patients that were alive at last follow-up and those that died of causes other than pancreatic cancer was censored as ending in a nonevent. All cases with missing information were included in proportional hazard ratio calculations after performing a sensitivity analysis which showed negligible effects of excluding missing data.

\section{Immunohistochemistry}

Immunohistochemical staining for CNKSR1 (mouse monoclonal antibody CNKSR1 (clone 46), Santa Cruz Biotechnology, TX, USA, \#sc-135,870; dilution 1:200) was performed on a Leica BOND-MAX autostainer (Leica Microsystems, IL, USA). Antigen retrieval was for $25 \mathrm{~min}$ with Bond Epitope Retrieval Solution 2 (Leica Biosystems Newcastle, UK, \#AR9640). Primary antibody was incubated for $30 \mathrm{~min}$ at room temperature. For detection the BondMax avidin biotin free polymer-based detection system (Bond Polymer Refine Detection \#DS9800) was used with diaminobenzidine as chromogen.

CNKSR1 coordinates signal transduction through interaction with proteins of distinct pathways in the cytoplasm [21]. Despite a fraction of tumors also showing concomitant expression of CNKSR1 in the nucleus, only cytoplasmic staining was scored for clinical correlative studies since if adopted as a prognostic test it would be more feasible to interpret only one parameter. Across all cases, staining for CNKSR1 was very uniform within each single tumor sample. Therefore, a simplified approach of scoring CNKSR1 immunohistochemistry was applied evaluating only staining intensities and not proportions of tumor cells stained [24, 25]. CNKSR1 expression was evaluated based on intensity semiquantitatively on a four-tier scale $(0=$ negative, $1=$ weak/background, 2 = moderate/positive, 3 = strongly positive). CNKSR1 shows minimal expression in lymphoid tissues according to RNA-Seq data and immunohistochemical staining from the Human Protein Atlas (Human Protein Atlas available from www.proteinatlas.org) [26]. Samples of lymph nodes and intratumoral lymphocytes were therefore used as negative controls.

Immunostaining for $\mathrm{p}$-ERK $1 / 2$ was developed using a rabbit monoclonal antibody (Cell Signaling, Cat\#4376) at 1:200 dilution. Staining was performed on the SEER Pancreas Cancer TMA only using standard IHC described above. With phosphorylation of extracellular-signalregulated kinase (ERK) inducing nuclear translocation staining was predominantly nuclear with a few cases also showing cytoplasmic staining. Scoring of p-ERK1/2 was done blinded to the CNKSR1 results using standard intensity scores above $(0=$ no staining, $1=$ weak staining, $2=$ moderate staining, $3=$ strong staining). In addition, the percentage of $\mathrm{p}$-ERK $1 / 2$ positive cells within an examined tumor core was scored and recorded as well.

Representative CNKSR1 staining patterns scored based on intensity of immunostaining in PDAC tissues are shown in Figs. 1 and 2. Representative p-ERK staining patterns scored based on intensity of immunostaining (no staining for p-ERK (score 0); weak p-ERK (score 1+), moderate p-ERK (score 2+), and strong p-ERK (score 3 +) staining) in Fig. 3. Staining intensities were grouped as dichotomous variables, defining scores $0-1$ as low and 2-3 as high expression levels [25]. Evaluation of staining was carried out independently by two pathologists (MM and MA) blinded to patients' outcome and pathological stage. Discrepant scores were discussed to reach an agreement.

\section{Statistical analysis}

Matched tumor and normal pancreatic tissues were compared using Wilcoxon matched-pairs signed rank test. Correlation of CNKSR1 and phospho-ERK expression levels was assessed by the Pearson's correlation coefficient test ( $r$; 2-tailed). Nuclear p-ERK expression levels in tumors were compared with the Mann Whitney $\mathrm{U}$ test (2-tailed) with cellular distribution of CNKSR1 (cytoplasmic only vs cytoplasmic and nuclear). Productlimit survival estimates were plotted using the KaplanMeier method with significance determined by log-rank test (PROC LIFETEST, SAS v 9.4, Cary, NC). Multivariate analysis was performed using Cox regression proportional hazard models (PROC PHREG, SAS v 9.4, Cary, NC) to estimate the risk of death among subjects with high CNKSR1 expression (reference group) compared to those with low CNKSR1 expression. A final model was developed using a stepwise variable selection process to adjust for gender, age, stage, grade, race, resection and radiation. Sensitivity analyses were performed after excluding cases 


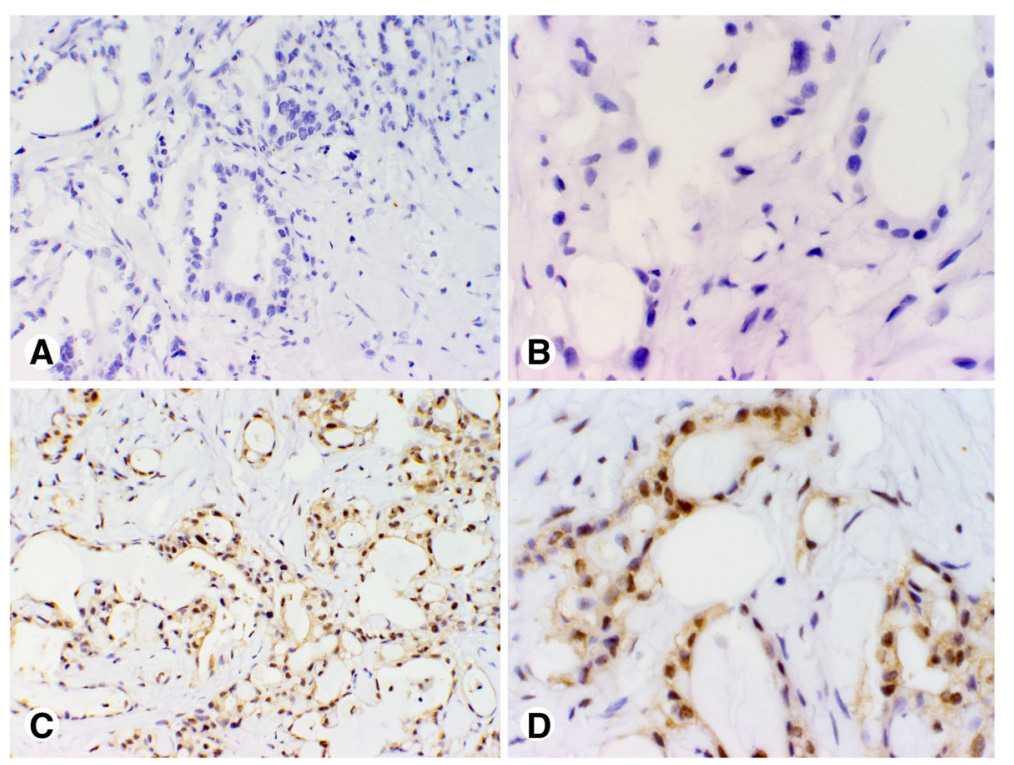

Fig. 1 Representative photomicrographs of pancreatic cancer tissue microarray (TMA) cores illustrating intensities of CNKSR1 immunohistochemical staining scored as low: a, b no staining for CNKSR1 (score 0); c, d weak CNKSR1 (score 1+) staining; only cytoplasmic staining was scored. Magnification: a, $\mathbf{c} \times 200 ; \mathbf{b}, \mathbf{d} \times 400$

with missing information on SEER stage (2), TNM stage (49) grade (1), resection (1) and radiation (4).

\section{Results}

\section{CNKSR1 is overexpressed in human pancreas cancer}

To examine if CNKSR1 expression is dysregulated in pancreas cancer we first compared CNKSR1 expression measured by intensity of immunostaining in 13 randomly chosen matched tumor and normal pancreatic tissues from the SEER Pancreatic Cancer TMA. Figure 4 shows elevated CNKSR1 protein expression levels in pancreatic tumors compared to matched, uninvolved controls $(p=0.004)$.

\section{CNKSR1 expression levels are heterogeneous in pancreatic adenocarcinoma}

Combining cases from all three cohorts, 6 cases showed no expression (5.0\%), 28 cases were scored as $1+(23.3 \%)$,

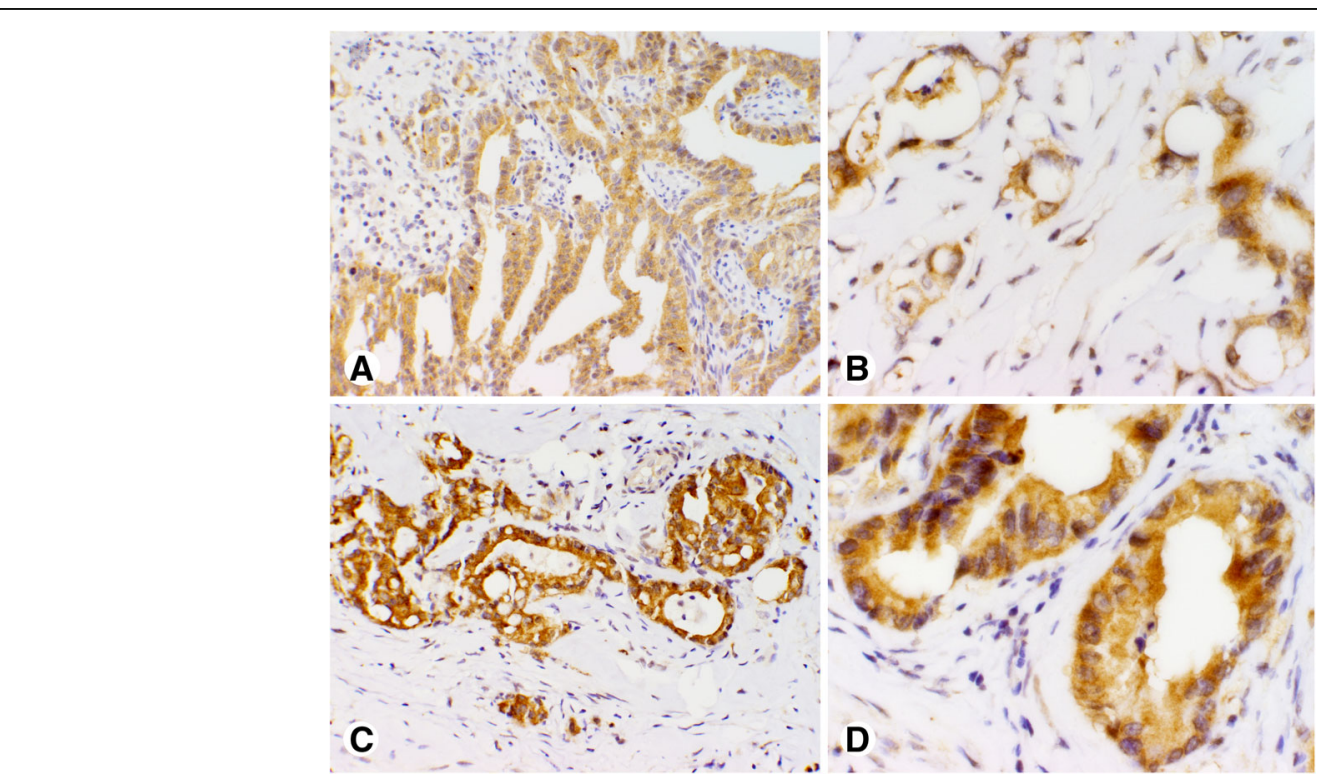

Fig. 2 Photomicrographs of pancreatic cancer tissue microarray (TMA) cores of high CNKSR1 immunohistochemical staining: $\mathbf{a}$, b positive for CNKSR1 (score 2+); c, d strongly positive for CNKSR1 (score 3+). Magnification: a, c $\times 200 ; \mathbf{b}, \mathbf{d} \times 400$ 


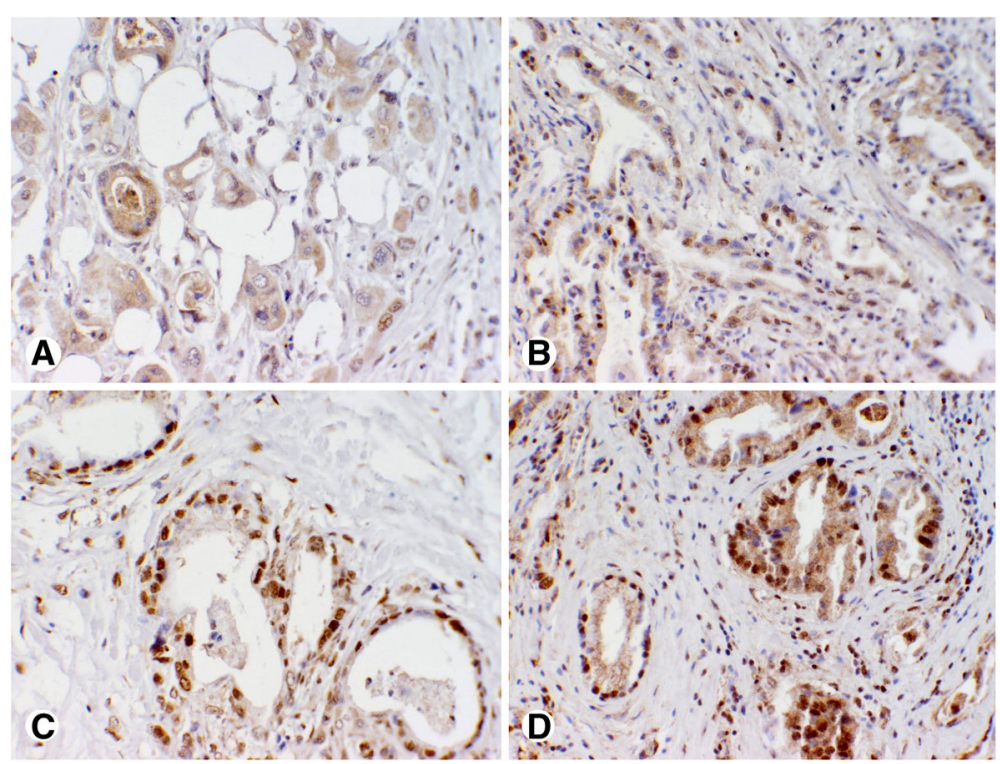

Fig. 3 Photomicrographs of pancreatic cancer tissue microarray (TMA) cores of $p$-ERK immunohistochemical staining: a no staining for $p$-ERK (score 0); b weak p-ERK (score 1+) staining; c moderate p-ERK (score 2+) staining; $\mathbf{d}$ strong p-ERK (score 3+) staining. Magnification: a-d: $\times 200$

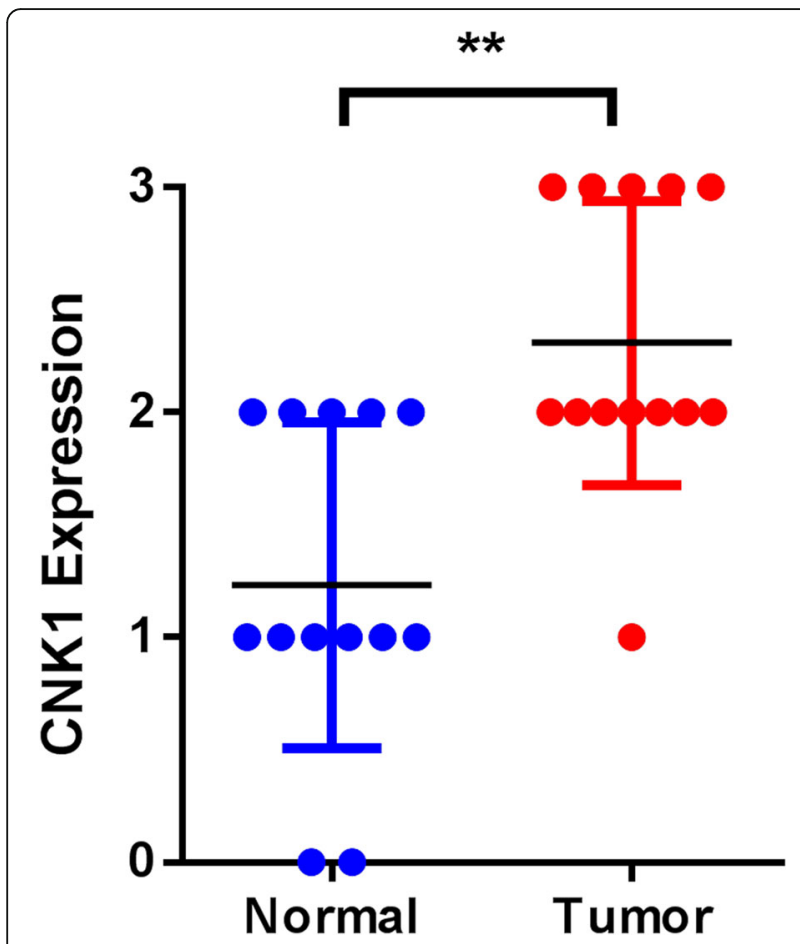

Fig. 4 CNKSR1 expression in matched tumor and normal pancreatic tissues. Increased CNKSR1 expression levels were observed in tumor tissues compared to matched normal tissue by Wilcoxon matchedpairs signed rank test $\left({ }^{* *} p=0.004\right)$
70 cases as $2+(58.3 \%)$, and 16 cases as $3+(13.3 \%)$ (Fig. 5a). To validate the expression pattern used for the clinical outcome associations in an independent cohort, 71 cases from a commercially available pancreatic cancer TMA and 47 cases from a TMA constructed at an outside institution were subject to the same CNKSR1 staining and reviewed by the same study pathologists (MM, MA). CNKSR1 low (0 and 1 + expression) versus CNKSR1 high $(2+, 3+)$ comprised 28.3 and $71.7 \%$ of cases in the study cohort and 44.1 and $55.9 \%$ of cases in the validation cohort suggesting similar expression patterns across the different arrays. In the study cohort $30 \%$ of cases also showed some degree of nuclear staining (Fig. 5b). Nuclear staining was lower than cytoplasmic expression levels. There was no association between cytoplasmic CNKSR1 expression levels $(0,1+$ vs $2+, 3+)$ and presence of nuclear CNKSR1 staining ( $p=0.22$ ) (Fig. 5b).

\section{CNKSR1 expression is prognostic of clinical outcome in pancreas cancer}

Table 1 presents demographic and clinical characteristics of the pancreatic cancer patient specimens used for clinical outcome associations (SEER, UMD, and NIH). Table 2 presents the demographics and tumor characteristics by CNKSR1 expression status. The majority of cases were 65 years of age or older at the time of diagnosis (59\%), regardless of CNKSR1 expression status. The gender distribution of cases was relatively balanced, including by CNKSR1 expression status. A higher proportion of CNKSR1 high cases had poorly differentiated grade 


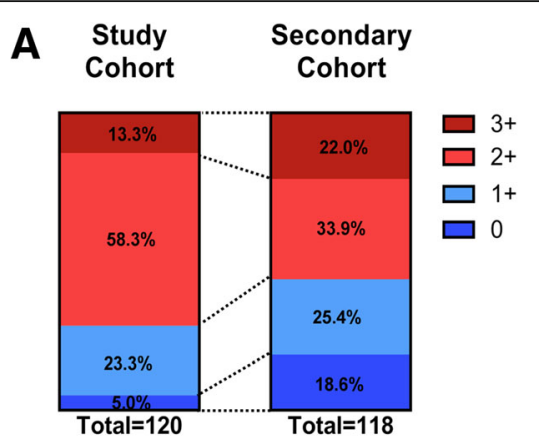

CNKSR1 Cellular Distribution
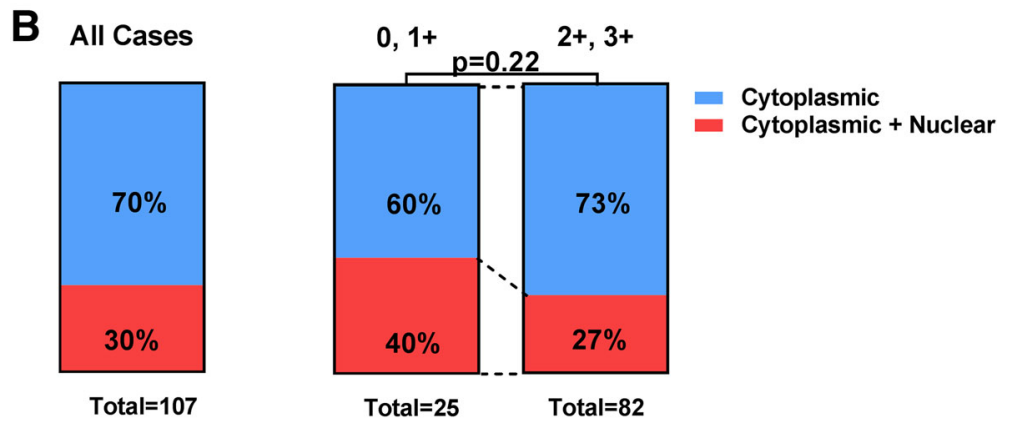

Fig. 5 a Comparison of CNKSR1 expression of study cohort and secondary validation cohort. b Cellular distribution pattern of CNKSR1 showed primarily cytoplasmic expression in pancreatic cancer specimens. Nuclear staining of CNKSR1 was not associated with cytoplasmic CNKSR1 expression levels $(0,1+$ vs $2+, 3+; p=0.22$; chi square test, 2-tailed)

tumors compared to CNKSR1 low cases (22\% vs 6\%; $p=0.04)$. CNKSR1 expression did not vary appreciably with stage or resection status.

Figure 6 presents survival probability by CNKSR1 expression. The 86 patients with high CNKSR1 expression survived a median of 14 months from diagnosis compared to 7.5 months for the 34 CNKSR1 low cases (logrank test, $p=0.03$ ).

Table 3 presents the unadjusted and adjusted hazard ratios for pancreatic cancer cases by CNKSR1 expression status. In the unadjusted model, cases with CNKSR1 low tumors had an increased risk of death compared to those with CNKSR1 high tumors with a hazard ratio (HR) of 1.61 (95\% CI: 1.06 to 2.46). In a model that adjusted for resection, TNM stage, age, gender, grade, receipt of radiation for localized or regional stage cancer, and race, the hazard ratio for CNKSR1 low compared to CNKSR1 high tumors was equal to 2.146 and the 95\% confidence interval ranged from 1.34 to 3.43 $(p=0.0014)$. Using SEER Summary Stage instead of partial TNM Staging revealed a similar hazard ratio (HR) of 1.91 (95\% CI: 1.20 to 3.05; data not shown). Other statistically significant variables in the model were, as expected and known from previous clinicopathological multivariate outcome models of pancreas cancer, no resection compared to any resection $(\mathrm{HR}=3.78,95 \% \mathrm{CI}$ : 2.09 to 6.85), TNM staging indicating regional lymph nodes $(\mathrm{HR}=1.89,95 \% \mathrm{Cl}: 1.21$ to 2.97$)$ and distant metastasis $(\mathrm{HR}=2.83,95 \% \mathrm{Cl}: 1.58$ to 5.07$)$, and age $65+(\mathrm{HR}=1.55$, 95\% CI:1.02 to 2.36 ). In sensitivity analyses, taking into account missing TNM staging data ( $\mathrm{N}, 49$ subjects), the association between low CNKSR1 expression and increased risk of death persisted (data not shown).

\section{CNKSR1 expression and outcome of patients following surgical resection}

Since the unfavorable course of low CNKSR1 expressing tumors suggests a more aggressive tumor biology, we investigated the possibility that the impact of resection on survival was diminished in this group. This analysis was limited to primary, non-metastatic tumor biopsies, since this represents the group where CNKSR1 status might be used in pre-operative decision-making. Patients with CNKSR1 low expression did not show an associated survival difference between resected patients and nonresected cases ( $p=0.3666$, Fig. 7$)$, while patients with high CNKSR1 expression did have a positive association between resection status and survival $(p<0.0001)$. Thus, CNKSR1 expression status might capture unfavorable tumor biology for surgical resection for pancreatic cancer, and may aid in pre-operative treatment selection. 
Table 1 Characteristics of patients included in the study

\begin{tabular}{|c|c|c|c|}
\hline & & All Patients $(N=120)$ & $\%$ \\
\hline \multicolumn{4}{|c|}{ Age group } \\
\hline & $<64$ & 49 & 41 \\
\hline & $65+$ & 71 & 59 \\
\hline \multicolumn{4}{|l|}{ Race } \\
\hline & White & 70 & 58 \\
\hline & Asian/PI & 35 & 29 \\
\hline & Black or Hispanic & 15 & 13 \\
\hline \multicolumn{4}{|l|}{ Gender } \\
\hline & Male & 58 & 48 \\
\hline & Female & 62 & 52 \\
\hline \multicolumn{4}{|l|}{ Grade } \\
\hline & Well Differentiated (1) & 65 & 54 \\
\hline & Moderately Diff. (2). & 34 & 28 \\
\hline & Poorly Diff. (3). & 21 & 18 \\
\hline \multicolumn{4}{|l|}{ Stage } \\
\hline & Localized & 12 & 10 \\
\hline & Regional & 69 & 58 \\
\hline & Distant & 39 & 33 \\
\hline \multicolumn{4}{|l|}{ TNM (N) } \\
\hline & Lymph Node Negative (NO) & 30 & 25 \\
\hline & Regional Lymph Nodes (N1) & 45 & 38 \\
\hline & Unknown (NX) & 45 & 38 \\
\hline \multicolumn{4}{|l|}{ TNM (M) } \\
\hline & No Metastasis (M0) & 77 & 64 \\
\hline & Distant Metastasis (M1) & 39 & 33 \\
\hline \multicolumn{4}{|l|}{ Resection } \\
\hline & Yes & 72 & 60 \\
\hline & No & 48 & 40 \\
\hline \multicolumn{4}{|l|}{ Radiation } \\
\hline & Yes & 30 & 25 \\
\hline & No & 90 & 75 \\
\hline \multicolumn{4}{|l|}{ Location } \\
\hline & Head & 67 & 56 \\
\hline & Body/Tail & 20 & 17 \\
\hline & Unknown & 33 & 28 \\
\hline
\end{tabular}

Phospho-ERK expression in relation to CNKSR1 expression pattern in the SEER pancreatic cancer TMA

To gain more insight into the mechanism of action and role of CNKSR1 in MAPK pathway regulation, we next measured expression levels of phospho-ERK in 86 matched cases of the SEER Pancreatic Cancer TMA previously evaluated and scored for CNKSR1 expression levels. As phosphorylation of the Extracellular-signal Regulated Kinase (ERK) induces nuclear translocation, p-ERK expression pattern was found to be predominantly nuclear, with only 14 cases (16\%) showing additional cytoplasmic staining and three cases (3\%) showing exclusive cytoplasmic staining without nuclear expression (Fig. 8a). There was significant heterogeneity in the degree of nuclear p-ERK levels, with 12 cases (14\%) staining weak (1+), 26 cases (29\%) moderate (2+), and 49 cases (57\%) strong $(3+)$. The presence of concomitant cytoplasmic staining was not associated with nuclear pERK1/2 expression levels (Fig. 8b). We next examined whether the cellular distribution of CNKSR1 was correlated with p-ERK expression levels scored by intensity of p-ERK immunostaining. Figure $8 \mathrm{C}$ shows nuclear $\mathrm{p}$-ERK expression levels $(0,1+, 2+, 3+))$ by CNKSR1 cellular distribution (cytoplasmic CNKSR1 expression only vs cytoplasmic and nuclear) in pancreas cancer specimens of the SEER Pancreatic Cancer TMA (Mann Whitney U test; $p=0.017)$. To test whether expression levels of the two proteins are correlated as well, including if a possible negative correlation between cytoplasmic CNKSR1 expression levels $(0,1+, 2+$, and $3+)$ and nuclear p-ERK levels (in \% of tumor cells with nuclear staining) exists, Pearson's correlation coefficient testing was employed (Fig. 8d). There was no correlation between cytoplasmic CNKSR1 expression levels and nuclear p-ERK expression levels $(r=0.24 ; p<0.05)$. There was a trend towards different overall outcome by stratification of patients by cellular distribution of p-ERK expression (nuclear vs nuclear and cytoplasmatic) (log rank test; $\mathrm{HR}=1.61$; $p=0.07)$. These finding suggest that cellular distribution of CNKSR1, rather than expression levels, might be involved in MAPK pathway and p-ERK regulation.

\section{Discussion}

In this study, we examined the expression levels of the scaffolding kinase CNKSR1 in pancreatic cancer surgical resection specimens and evaluated their impact on clinical outcome, including overall survival (OS). We found, in a limited set of matched normal and tumor specimens, that CNKSR1 expression is elevated in cancer tissue compared to normal uninvolved pancreas tissue. Elevated CNKSR1 expression correlated with improved survival as an independent prognostic marker using multivariate analysis, with patients in the low CNKSR1 expression group having a median OS that is nearly half that of patients with high CNKSR1 expression. In addition, we attempted to determine whether CNKSR1 status might affect the survival difference associated with resection in pancreatic cancer patients. If validated in a larger patient sample, such information might be useful in pre-operative decision-making.

CNKSR1 expression has not been previously shown to be associated with survival. This is in contrast to KSR1, the other major scaffolding protein of the MAPK/ERK pathway. KSR1, which tightly regulates 
Table 2 Demographics, tumor characteristics, and treatment by CNKSR1 expression status

\begin{tabular}{|c|c|c|c|c|c|c|}
\hline & & CNKSR1 Low $(N=34)$ & $\%$ & CNKSR1 High $(N=83)$ & $\%$ & $P$ \\
\hline \multirow[t]{3}{*}{ Age group } & & & & & & 0.72 \\
\hline & $<64$ & 13 & 38 & 36 & 42 & \\
\hline & $65+$ & 21 & 62 & 50 & 58 & \\
\hline \multirow[t]{4}{*}{ Race } & & & & & & 0.22 \\
\hline & White & 23 & 68 & 47 & 55 & \\
\hline & Asian/PI & 6 & 18 & 29 & 34 & \\
\hline & Black or Hispanic & 5 & 15 & 10 & 12 & \\
\hline \multirow[t]{3}{*}{ Gender } & & & & & & 0.82 \\
\hline & Male & 17 & 50 & 41 & 48 & \\
\hline & Female & 17 & 50 & 45 & 52 & \\
\hline \multirow[t]{4}{*}{ Grade } & & & & & & 0.04 \\
\hline & Well Differentiated (1) & 18 & 53 & 47 & 55 & \\
\hline & Moderately Diff. (2). & 14 & 41 & 20 & 23 & \\
\hline & Poorly Diff. (3). & 2 & 6 & 19 & 22 & \\
\hline \multirow[t]{4}{*}{ Stage } & & & & & & 0.86 \\
\hline & Localized & 4 & 12 & 8 & 9 & \\
\hline & Regional & 20 & 59 & 49 & 57 & \\
\hline & Distant & 10 & 29 & 29 & 34 & \\
\hline \multirow[t]{4}{*}{ TNM (N) } & & & & & & 0.70 \\
\hline & Lymph Node Negative (No) & 10 & 29 & 20 & 23 & \\
\hline & Regional Lymph Nodes (N1) & 11 & 32 & 34 & 40 & \\
\hline & Unknown (NX) & 13 & 38 & 32 & 37 & \\
\hline \multirow[t]{3}{*}{ TNM (M) } & & & & & & 0.11 \\
\hline & No Metastasis (M0) & 21 & 62 & 56 & 65 & \\
\hline & Distant Metastasis (M1) & 10 & 29 & 29 & 34 & \\
\hline \multirow[t]{3}{*}{ Resection } & & & & & & 0.87 \\
\hline & Yes & 20 & 59 & 52 & 60 & \\
\hline & No & 14 & 41 & 34 & 40 & \\
\hline \multirow[t]{3}{*}{ Radiation } & & & & & & 0.24 \\
\hline & Yes & 6 & 18 & 24 & 28 & \\
\hline & No & 28 & 82 & 62 & 72 & \\
\hline \multirow[t]{4}{*}{ Location } & & & & & & 0.11 \\
\hline & Head & 15 & 44 & 52 & 60 & \\
\hline & Body/Tail & 5 & 15 & 15 & 17 & \\
\hline & Unknown & 14 & 41 & 19 & 22 & \\
\hline
\end{tabular}

CNKSR1 expression status (low $=0,1$; high $=2,3$ staining intensity) for pancreatic cancer subjects by demographic, tumor characteristic and treatment option variables

MAPK/ERK signaling output, has been shown to be overexpressed in endometrial and colon carcinoma and high expression levels reported to be associated with decreased survival in breast cancer [14, 15, 27]. KSR1 has been shown to impact tumor biology, including in preclinical models of pancreas cancer, as well as response to chemotherapy [12]. To date, such information is largely lacking for CNKSR1, a binding partner and regulator of KSR1 and the MAPK pathway.
One of the main functions of the scaffold protein CNKSR1 is the regulation of other cancer-related signaling pathways integrating output to different intracellular signaling cascades upon cellular stimulation by extracellular cues [11, 12, 17-20]. In breast and cervical cancer cell models, a pro-oncogenic potential has been demonstrated through ERK-independent AKT-FoxO and NF-kappaB pathways [17, 19]. In a colorectal cancer cell model, CNKSR1 has been shown to facilitate pro-apoptotic 


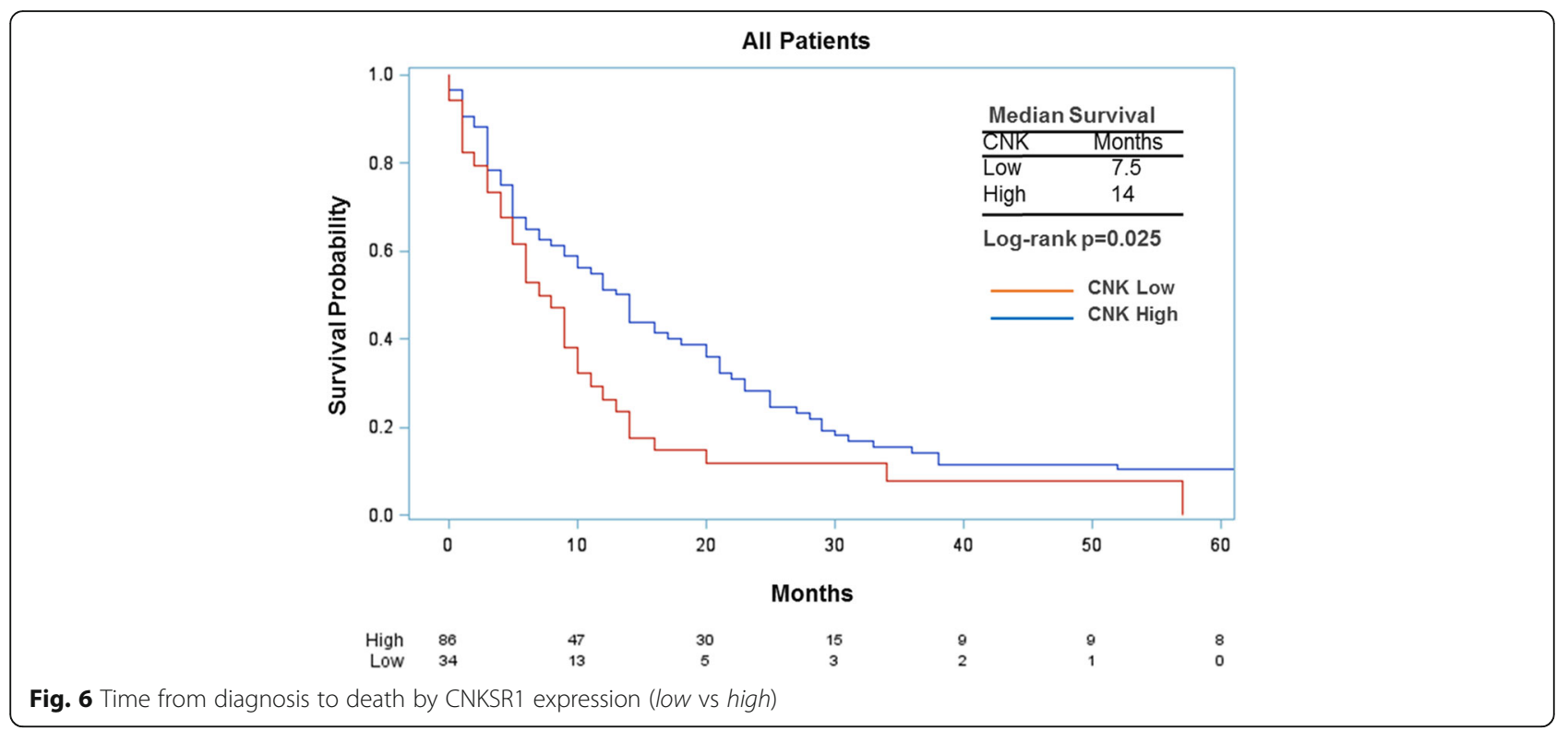

signaling via RASSF1A [18]. Importantly, a recent cellbased study expressing mutants of known phosphorylation sites of CNKSR1 in cancer cells identified sites linked to nuclear translocation with concomitant activation of MAPK pathway-driven serum response element (SRE) gene expression providing, for the first time, a direct correlation of cellular CNKSR1 distribution and MAPK pathway signaling output. [21] These findings are in line with the identified correlation of CNKSR1 cellular distribution (cytoplasmic only vs cytoplasmic and nuclear) and nuclear p-ERK expression levels observed in our study.
The finding that pancreatic tumors which have nuclear CNKSR1 expression in addition to the predominant cytoplasmic expression of CNKSR1 show higher nuclear p-ERK expression levels suggests that cellular localization, rather than absolute CNKSR1 expression levels, might be one of the mechanisms of CNKRS1-mediated control of MAPK pathway activity. In this regard, Fisher and colleagues showed in an elegant study with a set of different phosphomimetic mutant constructs that phosphorylation of Tyr 519 recruits CNKSR1 to the nucleus and phosphorylation of Tyr 26 enables CNKSR1 to activate MAPK

Table 3 Cox proportional hazard ratio estimates for death in pancreatic cancer subjects

\begin{tabular}{llll}
\hline Hazard Ratio Estimates for CNK Negative Status & & & \\
\hline Unadjusted model & Hazard Ratio & $95 \%$ Confidence Limits & $p$ value \\
CNK 0-1 & 1.611 & $1.057-2.457$ & 0.027 \\
Adjusted model & Hazard Ratio & $95 \%$ Confidence Limits & 0.0014 \\
CNK 0-1 & 2.146 & $1.341-3.434$ & $<.089-6.852$ \\
No Resection & 3.783 & $1.584-5.069$ & 0.0001 \\
Distant Metastasis (MO vs M1) & 2.834 & $1.206-2.972$ & 0.0004 \\
Regional Lymph Nodes (NO vs N1) & 1.893 & $1.018-2.359$ & 0.0055 \\
65+ Years at Diagnosis & 1.550 & $0.821-1.851$ & 0.041 \\
Male & 1.233 & $0.580-1.772$ & 0.26 \\
Moderately Differentiated & 1.014 & $0.508-1.356$ & 0.96 \\
Poorly Differentiated & 0.830 & $0.370-1.231$ & 0.45 \\
Non-Palliative Radiation & a \\
White & 0.674 & $0.288-1.298$ & 0.19 \\
Asian & 0.611 & $0.268-1.266$ & 0.20 \\
\hline
\end{tabular}

${ }^{a}$ Referent groups for respective variables were subjects with high CNKSR1 expression, those with resections, localized or regional stage cancer at diagnosis, females, cases less than 65 years of age, with localized or regional stage radiation therapy, Black or Hispanic race/ethnicity 

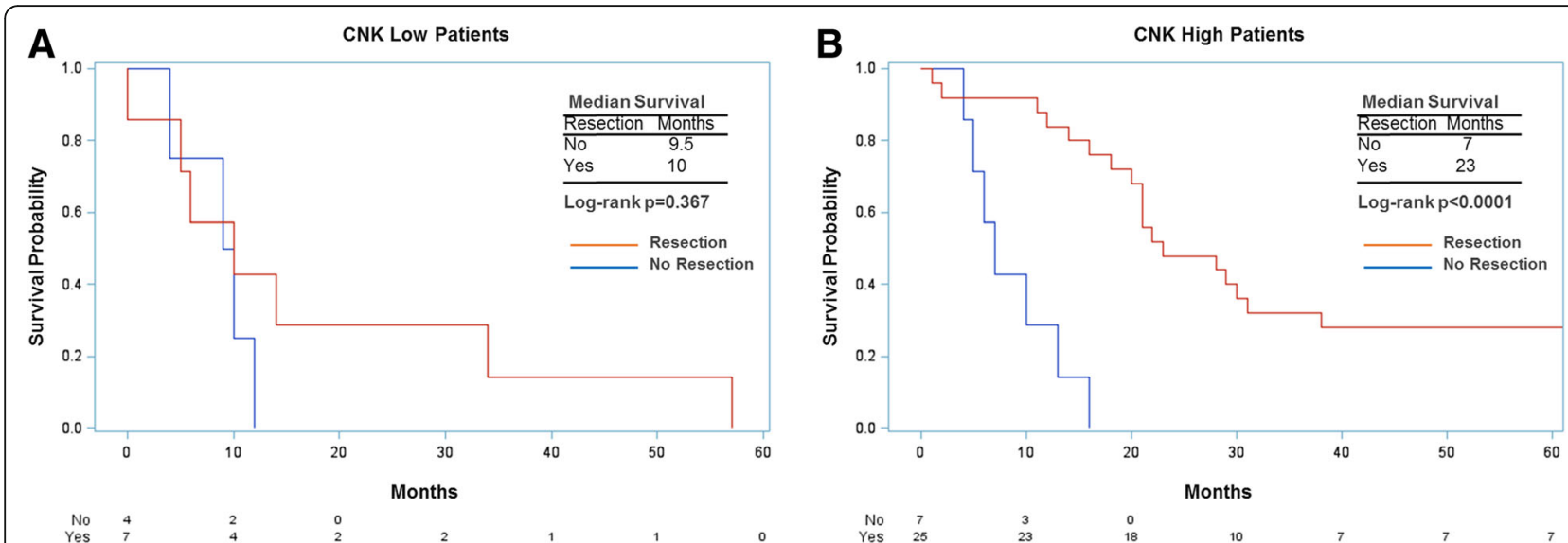

Fig. 7 Survival stratified by resection and CNKSR1 expression status. a Survival in limited subset of primary tumor biopsy specimens showing low CNKSR1 expression by resection status $(p=0.367)$. b Survival in patients with high CNKSR1 expression by resection status $(p<0.0001)$

pathway-governed serum response element (SRE) gene transcription [21]. Previous smaller correlative biomarker studies did show that increased nuclear p-ERK staining levels were associated with poorer survival in pancreas cancer [28, 29]. Such MAPK pathway regulation by nuclear translocation possibly induced by posttranslational modifications including phosphorylation of select sites of CNKSR1, appears in line with our findings of a correlation between the cellular distribution of CNKSR1 and nuclear p-ERK expression levels [30].

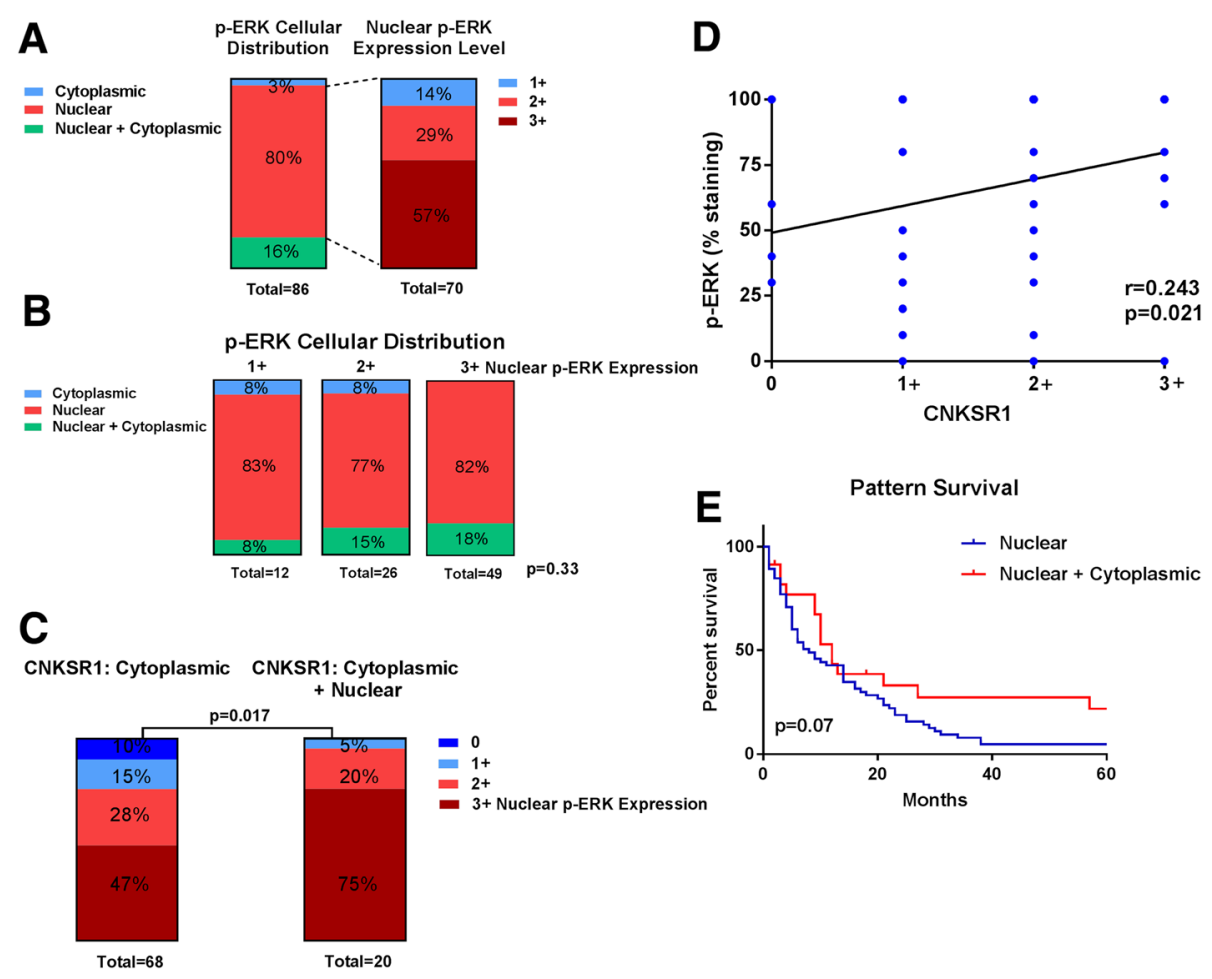

Fig. 8 Cellular distribution of CNKSR1 is associated with p-ERK expression levels in pancreatic cancer specimens. a p-ERK cellular distribution and nuclear expression levels (scored as 1+, 2+, and 3+) in 86 cases of the SEER Pancreatic Cancer TMA. b Nuclear expression levels of p-ERK (scored as $1+, 2+$, and $3+$ ) are not associated with presence of cytoplasmic p-ERK expression pattern. $\mathbf{c}$ Pancreatic cancer specimens with concomitant cytoplasmic and nuclear CNKSR1 expression have increased nuclear p-ERK staining (scored by 0, 1+, 2+, and 3+ intensity levels) compared to pancreatic cancers with cytoplasmic CNKSR1 staining only (Mann Whitney U test; 2-tailed). d Cytoplasmic CNKSR1 expression levels (scored semiquantitatively as $0,1+, 2+$, and $3+$ ) and nuclear p-ERK expression levels (scored as \% positive cells) (Pearson's correlation coefficient test; 2-tailed). e Kaplan-Meier survival analysis of pancreatic cancer cases from SEER Pancreatic Cancer TMA with p-ERK stratified by cellular distribution of p-ERK (nuclear vs nuclear and cytoplasmic) (log-rank; 2-tailed) 
While there have been more than 100 correlative biomarkers described investigating pancreatic cancer, there have been less than a dozen studies which correlated putative markers with survival outcome [31]. Similar to the hazard ratio (HR) estimates for death reported for CNKSR1 in this study, the majority of previously described prognostic biomarker studies reported HRs for death ranging from 1.5 to 4 [31]. Exceptions to these studies are, among others, the recently reported study of 13 putative pancreas cancer biomarkers from investigators at the Memorial Sloan-Kettering Cancer Center (MSKCC) describing two biomarkers with HRs exceeding ten [4]. It is also noteworthy that of the biomarkers reported to be associated with survival (reviewed by Winter et al. 2013), there are a number of proteins either indirectly or directly connected to MAPK/ERK signaling (Ras, ERBB2, Myc) [4, 31].

A limitation of this study was that the dataset did not include all clinical variables, such as complete TNM staging and chemotherapy treatment, and thus we could not evaluate all variables that might impact survival. While the HRs of known clinical (TNM system $\mathrm{N}$ and $\mathrm{M}$ stage) and pathological variables in the Cox proportional hazard ratio model yielded previously known associations with outcome, the T variable of the AJCC TNM staging system was not fully collected and only incompletely captured by the stage variable (localized vs regional). While this somehow limits direct comparability to previous studies, prospectively evaluated and validated nomograms on surgical outcome of pancreas cancer have shown that, by far, M and $\mathrm{N}$ stage, as captured in our study, are the strongest predictors of outcome [32, 33]. In addition, data regarding systemic therapy was not available, though nearly all cases were diagnosed prior to the routine use of FOLFIRINOX or Gemcitabine/nab-Paclitaxel.

We consider validation of the CNKSR1 expression pattern used for the correlative outcome studies in the two independent cohorts of pancreas cancer surgical specimens a strength of the study. Co-staining of matched SEER Pancreatic Cancer TMA specimens with p-ERK suggests a correlation between cellular distribution of CNKSR1 and nuclear p-ERK expression levels, possibly indicating regulation of MAPK pathway signaling less connected to absolute CNKSR1 expression levels. In addition, the SEER TMA studied has been used in multiple previous biomarker studies, several of which have been validated in additional larger cohorts [4, 22]. CNKSR1 may represent an additional prognostic marker in pancreatic cancer, and further validation in a larger cohort of recent patients is needed.

\section{Conclusion}

In this correlative tissue study including independent pancreatic cancer tissue microarrays from different sources, CNKSR1 expression was found to be an independent marker of patient outcome. We identified CNKSR1 low patients at high risk for disease progression suggesting that there might be distinct differences in tumor biology between CNKSR1 high and CNKRS1 low tumors. In CNKSR1 low expressing tumors, patients did not show any difference in survival when stratified by resection status whereas patients with high CNKSR1 expression levels who underwent resection had significantly improved outcome compared to non-resected patients in this group. Combination of CNKSR1 expression levels with current clinicopathological prognostic features might improve risk stratification and treatment selection.

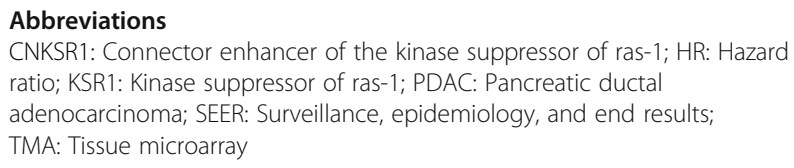

\section{Acknowledgments}

We would like to thank Dr. Zengfeng Wang (Laboratory of Pathology, NCl, $\mathrm{NIH)}$ for CNKSR1 immunohistochemical staining. This research was made possible through the National Institutes of Health $(\mathrm{NIH})$ Medical Research Scholars Program, a public-private partnership supported jointly by the $\mathrm{NIH}$ and generous contributions to the Foundation for the $\mathrm{NIH}$ from the Doris Duke Charitable Foundation, The American Association for Dental Research, The Howard Hughes Medical Institute, and the Colgate-Palmolive Company, as well as other private donors. For a complete list, please visit the Foundation website at: http://fnih.org/work/education-training-0/medicalresearch-scholars-program.

\section{Funding}

Intramural Research Program of the NIH, National Cancer Institute, Center for Cancer Research. The National Cancer Institute contracts for the participating SEER registries were N01-PC-35143 (lowa), N01-PC-35137 (Hawaii), and N01PC-35139 (Los Angeles).

\section{Availability of data and materials}

BioSample metadata are available in the NCBI BioSample database (http://www.ncbi.nlm.nih.gov/biosample/) under accession number SAMN05296738.

\section{Authors' contributions}

MA and MM performed pathology review of tissue specimens. RM and SA performed statistical analysis. The manuscript was written by HQ, TA, UR. The manuscript was reviewed and edited by MA, RM, and SH. Figures and tables were prepared by TA, RM, MA. All authors read and approved the final manuscript.

\section{Ethics approval and consent to participate}

The pancreatic TMA as well as pancreatic cancer biospecimens transferred under a Material Transfer Agreement (MTA) to NCl was approved by the Office of Human Subjects Research at the $\mathrm{NIH}$ and was found exempt from IRB review because it contained patient de-identified information.

\section{Consent for publication}

Not applicable.

\section{Competing interests}

The authors declare that they have no competing interests.

\section{Publisher's Note}

Springer Nature remains neutral with regard to jurisdictional claims in published maps and institutional affiliations. 


\section{Author details}

${ }^{1}$ Thoracic and Gastrointestinal Oncology Branch, Gastrointestinal Oncology Section, Investigator Center for Cancer Research, National Cancer Institute, Building 10 - Hatfield CRC, Room 4-5950, Bethesda, MD 20892, USA.

${ }^{2}$ Laboratory of Pathology, National Cancer Institute, Bethesda, MD, USA. ${ }^{3}$ Surveillance Informatics Branch, National Cancer Institute, Bethesda, MD, USA. ${ }^{4}$ Laboratory of Human Carcinogenesis, National Cancer Institute, Bethesda, MD, USA. ${ }^{5}$ Experimental Pathology Laboratory, Laboratory of Pathology, National Cancer Institute, Bethesda, MD, USA.

Received: 1 July 2016 Accepted: 12 July 2017

Published online: 21 July 2017

\section{References}

1. Rahib L, Smith BD, Aizenberg R, Rosenzweig AB, Fleshman JM, Matrisian LM. Projecting cancer incidence and deaths to 2030: the unexpected burden of thyroid, liver, and pancreas cancers in the United States. Cancer Res. 2014; 74(11):2913-21.

2. Siegel RL, Miller KD, Jemal A. Cancer statistics, 2016. CA Cancer J Clin. 2016; 66(1):7-30.

3. Evans DB, Varadhachary GR, Crane CH, Sun CC, Lee JE, Pisters PWT, Vauthey JN, Wang H, Cleary KR, Staerkel GA, et al. Preoperative gemcitabine-based chemoradiation for patients with resectable adenocarcinoma of the pancreatic head. J Clin Oncol. 2008:26(21):3496-502.

4. Winter JM, Tang LH, Klimstra DS, Brennan MF, Brody JR, Rocha FG, Jia XY, Qin LX, D'Angelica MI, DeMatteo RP, et al. A novel survival-based tissue microarray of pancreatic cancer validates MUC1 and Mesothelin as biomarkers. PLoS One. 2012;7(7):10

5. Jones S, Zhang XS, Parsons DW, Lin JCH, Leary RJ, Angenendt P, Mankoo P, Carter $\mathrm{H}$, Kamiyama $\mathrm{H}$, Jimeno $\mathrm{A}$, et al. Core signaling pathways in human pancreatic cancers revealed by global genomic analyses. Science. 2008; 321(5897):1801-6.

6. Lee J, Jang KT, Ki CS, Lim T, Park YS, Lim HY, Choi DW, Kaing WK, Park K, Park JO. Impact of epidermal growth factor receptor (EGFR) kinase mutations, EGFR gene amplilications, and KRAS mutations on survival of pancreatic adenocarcinoma. Cancer. 2007;109(8):1561-9.

7. Ogura T, Yamao K, Hara K, Mizuno N, Hijioka S, Imaoka H, Sawaki A, Niwa Y, Tajika M, Kondo S, et al. Prognostic value of K-ras mutation status and subtypes in endoscopic ultrasound-guided fine-needle aspiration specimens from patients with unresectable pancreatic cancer. J Gastroenterol. 2013;48(5):640-6.

8. Collisson EA, Sadanandam A, Olson P, Gibb WJ, Truitt M, Gu SD, Cooc J, Weinkle J, Kim GE, Jakkula L, et al. Subtypes of pancreatic ductal adenocarcinoma and their differing responses to therapy. Nat Med. 2011; 17(4):500-U140

9. Bailey P, Chang DK, Nones K, Johns AL, Patch AM, Gingras MC, Miller DK, Christ AN, Bruxner TJ, Quinn MC, et al. Genomic analyses identify molecular subtypes of pancreatic cancer. Nature. 2016:531(7592):47-52.

10. Kolch W. Coordinating ERKMMAPK signalling through scaffolds and inhibitors. Nat Rev Mol Cell Biol. 2005;6(11):827-37.

11. McKay MM, Morrison DK. Integrating signals from RTKs to ERK/MAPK. Oncogene. 2007;26(22):3113-21.

12. Kim M, Yan $Y$, Kortum RL, Stoeger SM, Sgagias MK, Lee $K$, Lewis RE, Cowan $\mathrm{KH}$. Expression of kinase suppressor of Ras1 enhances cisplatin-induced extracellular signal-regulated kinase activation and cisplatin sensitivity. Cancer Res. 2005;65(10):3986-92.

13. Zhang $\mathrm{H}, \mathrm{Koo} \mathrm{CY}$, Stebbing J, Giamas G. The dual function of KSR1: a pseudokinase and beyond. Biochem Soc Trans. 2013;41:1078-82.

14. Llobet D, Eritja N, Domingo M, Bergada L, Mirantes C, Santacana M, Pallares J, Macia A, Yeramian A, Encinas M, et al. KSR1 is Overexpressed in endometrial carcinoma and regulates proliferation and TRAlL-induced apoptosis by modulating FLIP levels. Am J Pathol. 2011;178(4):1529-43.

15. Wang L, Jiang CF, Li DM, Ge X, Shi ZM, Li CY, Liu X, Yin Y, Zhen LL, Liu LZ, et al. MicroRNA-497 inhibits tumor growth and increases chemosensitivity to 5fluorouracil treatment by targeting KSR1. Oncotarget. 2016;7(3):2660-71.

16. McKay MM, Ritt DA, Morrison DK. RAF inhibitor-induced KSR1/B-RAF binding and its effects on ERK cascade signaling. Curr Biol. 2011;21(7):563-8.

17. Fritz RD, Radziwill G. CNK1 promotes invasion of cancer cells through NFkappa B-dependent Signaling. Mol Cancer Res. 2010;8(3):395-406.

18. Rabizadeh S, Xavier RJ, Ishiguro K, Bernabeortiz J, Lopez-Hasaca M, Khokhlatchev A, Mollahan P, Pfeifer GP, Avruch J, Seed B. The scaffold protein CNK1 interacts with the tumor suppressor RASSF1A and augments RASSF1A-induced cell death. J Biol Chem. 2004:279(28):29247-54.

19. Fritz RD, Varga Z, Radziwill G. CNK1 is a novel Akt interaction partner that promotes cell proliferation through the Akt-FoxO signalling axis. Oncogene. 2010;29(24):3575-82

20. Cho HJ, Hwang YS, Mood K, Ji YJ, Lim J, Morrison DK, Daar IO. EphrinB1 interacts with CNK1 and promotes cell migration through c-Jun N-terminal kinase (JNK) activation. J Biol Chem. 2014;289(26):18556-68.

21. Fischer A, Brummer T, Warscheid B, Radziwill G. Differential tyrosine phosphorylation controls the function of CNK1 as a molecular switch in signal transduction. Biochim Biophys Acta. 2015;1853(11 Pt A):2847-55.

22. Takikita M, Altekruse S, Lynch CF, Goodman MT, Hernandez BY, Green M, Cozen W, Cockburn M, Saber MS, Topor M, et al. Associations between selected biomarkers and prognosis in a population-based pancreatic cancer tissue microarray. Cancer Res. 2009:69(7):2950-5.

23. Bosman FTCF, Hruban RH, Theise ND, editors. WHO classification of tumours of the digestive system. Geneva: WHO Press; 2010.

24. Bösmüller $H$, Fischer $A$, Pham DL, Fehm T, Capper D, von Deimling A, Bonzheim I, Staebler A, Fend F. Detection of the BRAF V600E mutation in serous ovarian tumors: a comparative analysis of immunohistochemistry with a mutation-specific monoclonal antibody and allele-specific PCR. Hum Pathol. 2013:44(3):329-35.

25. Ormanns S, Altendorf-Hofmann A, Jackstadt R, Horst D, Assmann G, Zhao Y, Bruns C, Kirchner T, Knosel T. Desmogleins as prognostic biomarkers in resected pancreatic ductal adenocarcinoma. Br J Cancer. 2015;113(10):1460-6.

26. Uhlén $M$, Fagerberg $L$, Hallström BM, Lindskog C, Oksvold P, Mardinoglu A, Sivertsson $\AA$, Kampf C, Sjöstedt E, Asplund A, et al. Tissue-based map of the human proteome. Science. 2015;347(6220).

27. Stebbing J, Zhang H, Xu Y, Lit LC, Green AR, Grothey A, Lombardo Y, Periyasamy $M$, Blighe $K$, Zhang $W$, et al. KSR1 regulates BRCA1 degradation and inhibits breast cancer growth. Oncogene. 2015;34(16):2103-14.

28. Chadha KS, Khoury T, Yu J, Black JD, Gibbs JF, Kuvshinoff BW, Tan D, Brattain MG, Javle MM. Activated Akt and Erk expression and survival after surgery in pancreatic carcinoma. Ann Surg Oncol. 2006;13(7):933-9.

29. Javle MM, Gibbs JF, Iwata KK, Pak Y, Rutledge P, Yu J, Black JD, Tan D, Khoury T. Epithelial-mesenchymal transition (EMT) and activated extracellular signal-regulated kinase ( $\mathrm{p}$-Erk) in surgically resected pancreatic cancer. Ann Surg Oncol. 2007;14(12):3527-33.

30. Fischer A, Brummer T, Warscheid B, Radziwill G. Differential tyrosine phosphorylation controls the function of CNK1 as a molecular switch in signal transduction. Biochim Biophys Acta (BBA) - Mol Cell Res. 2015; 1853(11, Part A):2847-55.

31. Winter JM, Yeo CJ, Brody JR. Diagnostic, prognostic, and predictive biomarkers in pancreatic cancer. J Surg Oncol. 2013;107(1):15-22.

32. Brennan MF, Kattan MW, Klimstra D, Conlon K. Prognostic Nomogram for patients undergoing resection for Adenocarcinoma of the pancreas. Ann Surg. 2004;240(2):293-8.

33. Ferrone CR, Kattan MW, Tomlinson JS, Thayer SP, Brennan MF, Warshaw AL. Validation of a Postresection pancreatic Adenocarcinoma Nomogram for disease-specific survival. J Clin Oncol. 2005;23(30):7529-35.

\section{Submit your next manuscript to BioMed Central and we will help you at every step:}

- We accept pre-submission inquiries

- Our selector tool helps you to find the most relevant journal

- We provide round the clock customer support

- Convenient online submission

- Thorough peer review

- Inclusion in PubMed and all major indexing services

- Maximum visibility for your research

Submit your manuscript at www.biomedcentral.com/submit
) Biomed Central 